\title{
STREPTOCOCCAL AGGLUTININS IN CHRONIC INFECTIOUS ARTHRITIS ${ }^{1}$
}

\author{
By EDITH E. NICHOLLS AND WENDELL J. STAINSBY
}

(From the Second (Cornell) Medical Division and the Pathological Laboratories of Bellevue Hospital, the Cornell Clinic, and the Department of Medicine, Cornell University Medical College, New York)

(Received for publication December 12, 1930)

The development of specific agglutinins in the course of acute or chronic infections has long been recognized as one of the most constant and characteristic of the phenomena which follow bacterial invasion of the animal organism. Indeed, this biologic reaction is such a well-nigh universal concomitant of infection that it has come into wide use clinically as a diagnostic measure.

In a previous paper, the writers (1), in collaboration with R. L. Cecil, have reported the isolation of streptococci from the blood and joints of a high percentage of patients with chronic infectious arthritis. Eighty-three and three-tenths per cent of the streptococcal strains thus isolated were culturally identical and were designated as "typical strains." Somewhat to our surprise, these "typical strains" were cross agglutinated by homologous immune rabbit serums. These observations naturally led to a search for streptococcal agglutinins in the serums of patients with chronic infectious arthritis, and the present communication embraces the results obtained in this investigation.

\section{METHODS}

1. Aggluiination tests. The antigen consisted of a 24-hour growth of a strain of streptococcus in beef heart infusion broth ( $\mathrm{pH}$ 7.6). Five-tenths cubic centimeter of the bacterial antigen was added in turn to a series of agglutination tubes each containing $0.5 \mathrm{cc}$. of the diluted serum. The dilutions were made in plain broth and the final

${ }^{1}$ This investigation was supported in part by the Committee for the Encouragement of Medical Research. 
dilutions were $1: 20,1: 40,1: 80,1: 160,1: 320,1: 640,1: 1,280,1: 2,560$ and 1:5,120. The controls consisted of normal serum and broth without serum. The tubes were heated in a water bath at $56^{\circ} \mathrm{C}$. for 2 hours, placed in the refrigerator overnight and read the following morning. The highest dilution in which definite agglutination was observed was recorded as the agglutination titer. Most of the agglutination tests were carried out by the operator without any knowledge of the identity of the serums.

At times there was a tendency for the cultures to become granular. This difficulty was overcome by successive passages of the streptococcal strain through blood broth for several days before finally transferring it to plain broth.

2. Absorption tests. Five tenths cubic centimeter of a 1:5 dilution of the serum in broth was added to the sediment from $150 \mathrm{cc}$. of a 24hour.broth culture of the streptococcus. The mixture was thoroughly shaken and placed for 2 hours in a water bath at $37^{\circ} \mathrm{C}$. where it received further shaking at frequent intervals. The mixture was then placed in the refrigerator overnight, and in the morning it was centrifugalized and the supernatant fluid removed. Agglutination tests were then carried out with the absorbed serum, using the same dilutions as in the original reactions. Control tests were carried out with unabsorbed serum. All the tubes were then heated for 2 hours in a water bath at $56^{\circ} \mathrm{C}$. Readings were made the following morning.

\section{Chronic infectious arthritis}

The type of patient investigated presented the syndrome generally known as chronic infectious arthritis or rheumatoid arthritis, namely, a chronic progressive polyarthritis with swelling and often with some deformity and ankylosis of the joints affected. The fusiform swelling of the fingers is a characteristic physical sign.

Agglutination reactions were carried out with the serums from 110 cases of chronic infectious arthritis against "typical strain" streptococci which had previously been recovered from the blood or joints of patients with this disease. Various "typical strains," but especially strain AB13, were used in these studies. Of the 110 serums examined, 103 , or 93.6 per cent, showed definite agglutination with "typical 
strain" streptococci to a titer of $1: 640$ or more (table 1, chart 1). Two of the remaining 7 serums were from patients who had had arthritic symptoms for only 8 and 60 days respectively.

\section{Controls}

Agglutination reactions against "typical strain" streptococci were carried out in the same way with the serums of 218 normal persons or patients with other diseases. It was considered advisable to divide the controls into several groups, namely, rheumatic fever, degenerative

TABLE 1

Titer of agglutination reactions of "typical strains" with patients' serums

\begin{tabular}{|c|c|c|c|c|c|c|c|c|c|c|c|}
\hline \multirow{2}{*}{ Disease } & \multirow{2}{*}{ 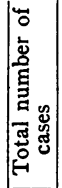 } & \multirow{2}{*}{ 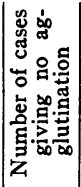 } & \multicolumn{9}{|c|}{ Dilutions of serum* } \\
\hline & & & $\stackrel{\text { ㄱ. }}{\underset{-}{*}}$ & 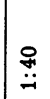 & $\begin{array}{l}\stackrel{\circ}{\infty} \\
\stackrel{-}{*}\end{array}$ & $\stackrel{8}{\stackrel{\bullet}{ت}}$ & 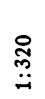 & 㐘 & 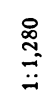 & $\begin{array}{l}\stackrel{8}{\circ} \\
\stackrel{\sim}{\pi} \\
\end{array}$ & 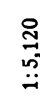 \\
\hline Chronic infectious arthritis. . & 110 & 1 & 1 & 1 & 1 & 1 & 2 & 17 & 23 & 39 & 24 \\
\hline Acute rheumatic fever. ..... & 79 & 51 & 3 & 12 & 10 & 2 & 1 & 0 & 0 & 0 & 0 \\
\hline Degenerative arthritis...... & 16 & 9 & 3 & 2 & 2 & 0 & 0 & 0 & 0 & 0 & 0 \\
\hline $\begin{array}{l}\text { Acute tonsillitis, acute bron- } \\
\text { chitis............. }\end{array}$ & 8 & 0 & 0 & 0 & 4 & 1 & 1 & 1 & 0 & 1 & 0 \\
\hline Other febrile diseases. . . . . . . & 27 & 15 & 2 & 3 & 3 & 2 & 1 & 1 & 0 & 0 & 0 \\
\hline Other non-febrile diseases... . & 41 & 22 & 4 & 6 & 5 & 2 & 0 & 1 & 1 & 0 & 0 \\
\hline Normal people.......... & 47 & 37 & 1 & 4 & 4 & 1 & 0 & 0 & 0 & 0 & 0 \\
\hline Healthy rabbits........ & 6 & 6 & 0 & 0 & 0 & 0 & 0 & $\mathbf{0}$ & 0 & 0 & 0 \\
\hline
\end{tabular}

* The numerals under the various dilutions indicate the number of cases with the limit of agglutination at that dilution.

arthritis, acute tonsillitis and acute bronchitis, other febrile diseases, other non-febrile diseases and normal individuals. The serums of 6 healthy rabbits were also studied for streptococcal agglutinins.

The serums of 79 patients with acute rheumatic fever were examined for specific agglutinins against the "typical strain" of streptococcus. Of these, 51, or 64 per cent, failed to show any agglutination, while none of them gave an agglutination to a titer higher than 1:320 (table 1, chart 1).

The next control group consisted of 16 cases of degenerative arthritis. Care was taken to select patients who showed degenerative 
arthritic changes only; none of the joints showed soft tissue swelling and $x$-rays revealed the characteristic hypertrophic changes in the bones. Of these 16 cases, 9 , or 56.3 per cent, showed no agglutination whatever with "typical strain" streptococci; the remaining 7 , gave very weakly positive reactions, 3 at a titer of $1: 20,2$ at a titer of 1:40,

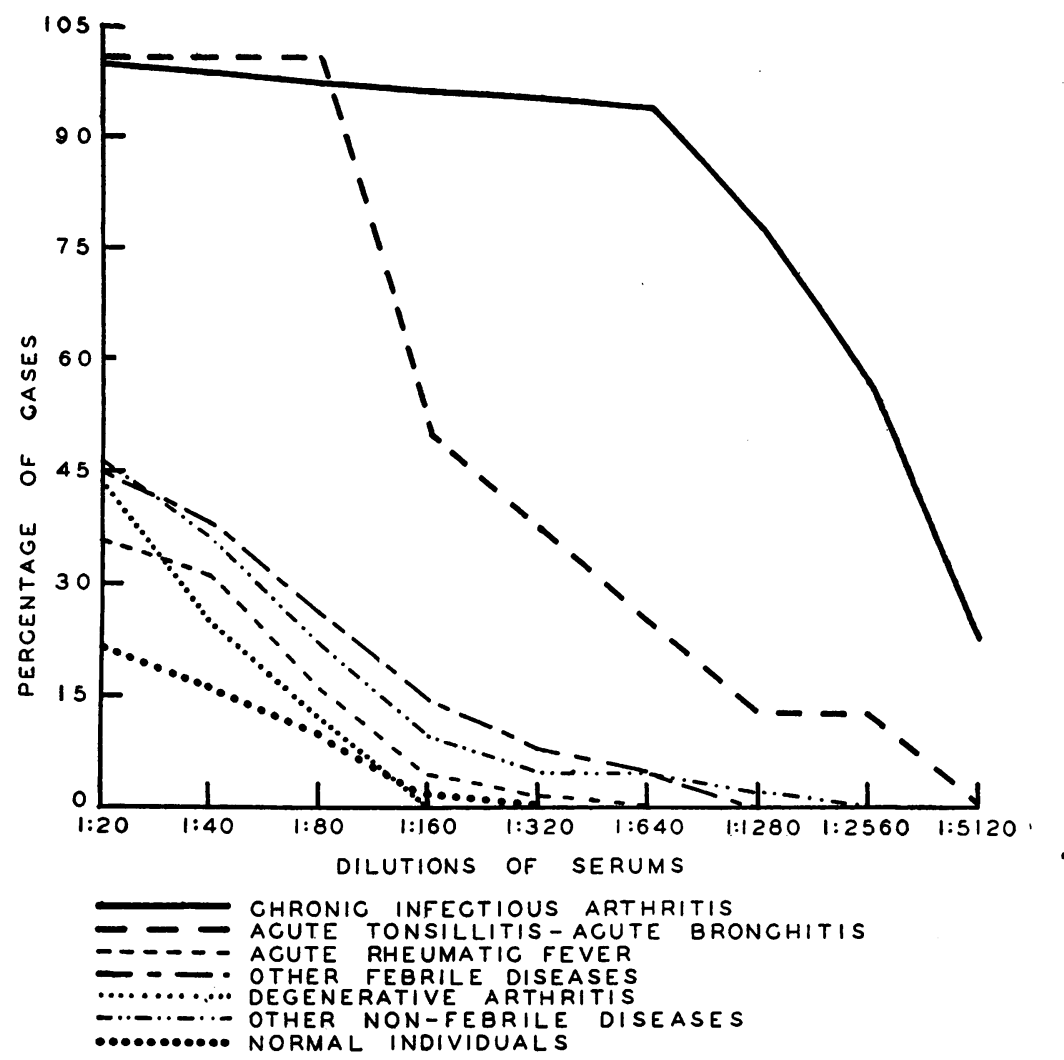

Chart 1. Total Agglutinations with Patients' Serums Against "Typical StranNs"

and 2 at a titer of 1:80 (table 1, chart 1). Probably these weakly positive reactions have no significance. In view of the fact that, in our experience, streptococci have never been recovered from the blood and joints of patients with degenerative arthritis, the absence of streptococcal agglutinins in the latter group of cases is quite consistent, 
and in striking contrast to the strong reactions obtained in cases of chronic infectious arthritis.

An interesting control group consisted of 8 patients suffering.from acute tonsillitis or acute bronchitis. The serum of one of these, a case of acute bronchitis, agglutinated with "typical strains" to a titer of $1: 2,560$, while the lowest agglutination titer recorded for this group of patients was 1:80 (table 1, chart 1). The serums of some of these patients gave agglutination reactions comparable to the serums of patients with chronic infectious arthritis, although the average titer for the upper respiratory infections was considerably lower. These observations were particularly interesting in view of the frequent association of infections of the upper respiratory tract with chronic infectious arthritis.

Twenty-seven patients with febrile conditions other than rheumatic fever and acute upper respiratory infections were tested for specific agglutinins against "typical strains" of streptococci. This group included cases of jaundice, pleurisy with effusion, acute nephritis, and pneumonia. The serum of only one of these, a patient with acute nephritis, gave an agglutination titer as high as 1:640; 15, or 55 per cent, showed no agglutination whatever, while the remaining cases showed only weakly positive reactions (table 1 , chart 1 ). The negative or weakly positive results obtained from this group of controls would appear to indicate that the agglutination observed in patients with chronic infectious arthritis cannot be looked upon as a physical phenomenon indicative of infection in general but as a specific reaction to streptococcus infection.

The serum of 41 patients with non-febrile diseases other than arthritis were studied in the same way. This group consisted of patients with new growths, hypertension, and other non-febrile diseases. Only 2 of the 41 cases gave an agglutination of $1: 640$ or higher; 22, or 53.7 per cent, showed no agglutination, while the remainder gave positive agglutinations only in the lower dilutions (table 1, chart 1).

The serums of 47 normal persons were studied in the same manner; 37 , or 78.7 per cent, failed to show any agglutination whatever with the "typical strain" of streptococcus. The highest agglutination titer recorded for this group was 1:160, a titer which has little, if any, significance (table 1, chart 1). 
The serums of 6 healthy rabbits failed to show any agglutination with "typical strain" cultures.

\section{Chronic polyarthritis following rheumatic fever}

During the course of this investigation, the serums of 3 patients with chronic polyarthritis following rheumatic fever were studied for the presence of streptococcal agglutinins. All 3 patients gave histories of one or more attacks of rheumatic fever. Clinically these three cases presented a picture indistinguishable from that of chronic infectious arthritis except for the presence of endocarditis presumably rheumatic. The agglutination titer with "typical strain" cultures was $1: 80$ in one case, and entirely negative in the other two cases. As will be shown in a subsequent communication, these patients showed strongly positive agglutination reactions with a streptococcus recovered from the blood of a patient with acute rheumatic fever.

\section{Disappearance of streptococcal agglutinins after recovery from chronic infectious arthritis}

In 3 patients who recovered from chronic infectious arthritis, a comparison of the agglutination reactions with the "typical strain" of streptococcus during the course of the disease was made with the reactions in the same patients after recovery from the infection.

A brief summary of the 3 cases is presented:

M. P., female, aged 27, chronic infectious arthritis of 2 years' duration. Agglutination titer with "typical strain" $1: 1,280$. Tonsillectomy performed, following which patient became free from symptoms. Three weeks after tonsillectomy the agglutination titer was $1: 320$, one year after, the agglutination test was entirely negative.

M. D., female, aged 30, chronic infectious arthritis of 3 months' duration; agglutination titer with "typical strain" 1:1,280. Tonsillectomy performed, following which patient completely recovered from arthritis. Three weeks after tonsillectomy agglutination titer was $1: 40 ; 3$ months after, the agglutination titer was entirely negative.

S. W., male, aged 50, chronic infectious arthritis of 3 months' duration; agglutination titer with "typical strain" $1: 2,560$. Tonsillectomy performed, following which patient became free from active disease. Three months after tonsillectomy agglutination test was entirely negative. 


\section{Stability of the streptococcal agglutinins}

Agglutination reactions were performed on the serums of patients suffering from chronic infectious arthritis with "typical strains," at approximately weekly intervals up to 90 days, in order to determine whether or not the serums retained their agglutinins for these streptococci over an appreciable length of time. During this period the serums were kept in a refrigerator at almost freezing temperature. It was found that the agglutinating property did not diminish perceptibly during the entire 90 days.

\section{Streptococcal agglutinins in joint fluids}

Agglutination reactions with "typical strains" were carried out on the joint fluids of 3 patients with chronic infectious arthritis. The technic for testing the joint fluids was similar in every respect to that employed for serums. The agglutination titers were surprisingly high, $1: 640,1: 2,560$, and 1:5,120, respectively. The only control joint fluids available were from 4 patients with acute rheumatic fever. The agglutination reaction in each of the controls was entirely negative.

\section{Specificity of the agglutination reactions}

It has already been shown that the serums of patients with chronic infectious arthritis agglutinate to a high titer with "typical strains." In order to check the specificity of these reactions, agglutination tests were performed with the serums of patients with chronic infectious arthritis against various pathogenic organisms. This work was controlled with the serums of patients having rheumatic fever, subacute bacterial endocarditis, and acute tonsillitis and with the serums of normal individuals (table 2).

As was expected, "typical strain" AB13 showed the usual high titers of agglutination with the serums of patients with chronic infectious arthritis. Much to our surprise, a Streptococcus hemolyticus from a case of scarlet fever and a Streptococcus hemolyticus from a case of erysipelas gave agglutination titers with the arthritic serums comparable to that given by the "typical strain" streptococcus, although on the average the end reactions were at lower dilutions. A rheumatic fever strain 


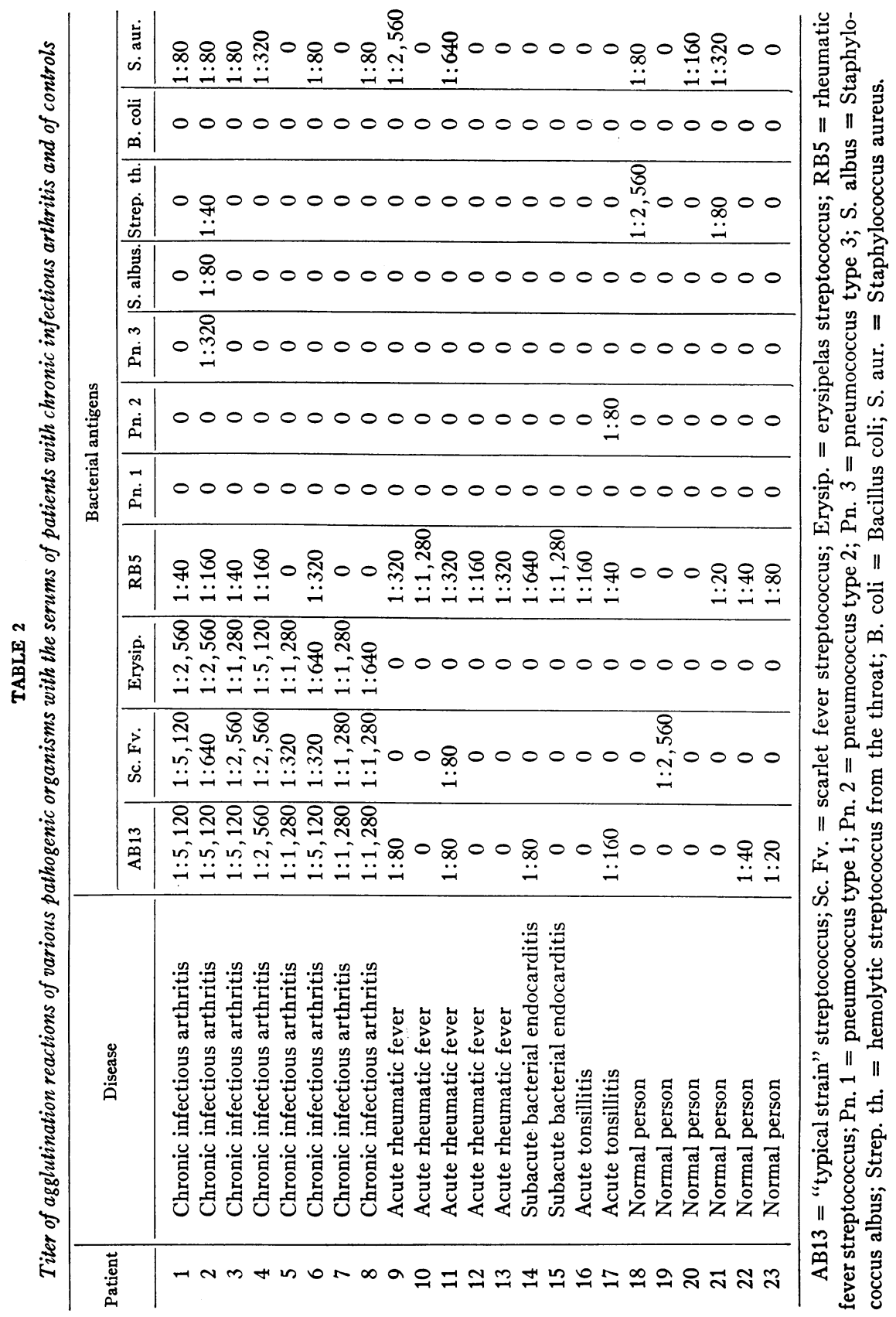


of Streptococcus viridans (RB5) showed weakly positive reactions with arthritic serums, but the titers were only slightly higher than those given by this streptococcus against normal serums. With the exception of the streptococci from chronic infectious arthritis, scarlet fever, and erysipelas, the various bacterial antigens tested showed only insignificant reactions. The serums from patients with other infections and from normal persons gave agglutination reactions of little importance except in the case of the rheumatic fever strain RB5 which showed strong agglutination reactions with the serums from cases of rheumatic fever and subacute bacterial endocarditis.

The agglutination tests with the serums of patients having chronic infectious arthritis against "typical strain" AB13, the scarlet fever streptococcus and the erysipelas streptococcus were controlled by direct absorption tests. These were performed by absorbing each of the arthritic serums with cultures of each of the three hemolytic streptococci. Agglutination tests were then carried out with the absorbed serums against their absorbing strains. Unabsorbed serums were used as controls. Complete or almost complete absorption was effected in each case.

The nature of the agglutinins in the serums of patients with chronic infectious arthritis was further studied by cross-absorption reactions with the serums of 2 patients with chronic infectious arthritis and the serum of a rabbit which had been previously immunized with the "typical strain" AB13. Each of the three serums was in turn subjected to absorption tests by the "typical strain" streptococcus AB13, the scarlet fever streptococcus and the erysipelas streptococcus. Agglutination tests were then carried out with the cultures of each of the three hemolytic streptococci on each of the absorbed serums. Complete or almost complete absorption was effected in each case (table 3). The cross-absorption tests indicate the antigenic relationship of "typical strain" streptococci to the hemolytic streptococci from cases of scarlet fever and erysipelas.

Finally, it seemed important to perform additional agglutination tests with arthritic serums against hemolytic streptococci from patients who gave no history of arthritis, scarlet fever, or erysipelas. Eight such strains were selected (table 4). Two of these strains, both recovered from throat cultures, showed an agglutination titer as high 
TABLE 3

Agglutination and absorption reactions with the serums of two patients with chronic infectious arthritis and of one immune rabbit against hemolytic streptococci from chronic infectious arthritis, scarlet fever and erysipelas

\begin{tabular}{|c|c|c|c|c|}
\hline \multirow{2}{*}{ Serums } & \multirow{2}{*}{ Absorbing strains } & \multicolumn{3}{|c|}{ Bacterial antigens } \\
\hline & & $\mathrm{AB} 13$ & S. F. 1 & E. 1 \\
\hline R. W. & $\begin{array}{l}\text { Unabsorbed } \\
\text { AB13 } \\
\text { S. F. } 1 \\
\text { E. } 1\end{array}$ & $\begin{array}{c}1: 1,280 \\
0 \\
0 \\
1: 20\end{array}$ & $\begin{array}{c}1: 640 \\
0 \\
0 \\
0\end{array}$ & $\begin{array}{c}1: 320 \\
0 \\
0 \\
0\end{array}$ \\
\hline L. M. & $\begin{array}{l}\text { Unabsorbed } \\
\text { AB13 } \\
\text { S. F. } 1 \\
\text { E. } 1\end{array}$ & $\begin{array}{c}1: 5,120 \\
0 \\
0 \\
1: 20\end{array}$ & $\begin{array}{c}1: 640 \\
0 \\
0 \\
0\end{array}$ & $\begin{array}{c}1: 640 \\
0 \\
0 \\
0\end{array}$ \\
\hline Rabbit 177 & $\begin{array}{l}\text { Unabsorbed } \\
\text { AB13 } \\
\text { S. F. } 1 \\
\text { E. } 1\end{array}$ & $\begin{array}{c}1: 5,120 \\
0 \\
0 \\
0\end{array}$ & $\begin{array}{c}1: 640 \\
1: 20 \\
0 \\
0\end{array}$ & $\begin{array}{c}1: 1,280 \\
0 \\
1: 20 \\
1: 20\end{array}$ \\
\hline
\end{tabular}

AB13 = "typical strain" steptococcus; S. F. 1 = scarlet fever streptococcus; E. 1 = erysipelas streptococcus; R. W. and L. M. = patients with chronic infectious arthritis; rabbit 177 = rabbit immunized against strain AB13.

\section{TABLE 4}

Titer of agglutination reactions of various hemolytic streptococci with the serums of patients with chronic infectious arthritis

\begin{tabular}{c|c|c|c|c|c|c|c|c|c|c|c}
\hline Patient & \multirow{2}{*}{ AB13 } & Sc. Fv. & Erysip. & $\begin{array}{c}\text { Throat } \\
\text { H.S. } 1\end{array}$ & $\begin{array}{c}\text { Throat } \\
\text { H.S. 2 }\end{array}$ & $\begin{array}{c}\text { Throat } \\
\text { H.S. } 3\end{array}$ & $\begin{array}{c}\text { Throat } \\
\text { H.S. } 4\end{array}$ & Tonsil & Tooth & Stool & $\begin{array}{c}\text { Gastric } \\
\text { ulcer }\end{array}$ \\
\hline 24 & $1: 5,120$ & $1: 1,280$ & $1: 160$ & $1: 80$ & $1: 20$ & $1: 80$ & 0 & 0 & 0 & $1: 80$ & $1: 20$ \\
25 & $1: 640$ & $1: 1,280$ & $1: 160$ & 0 & 0 & $1: 80$ & 0 & $1: 160$ & 0 & 0 & $1: 20$ \\
26 & $1: 640$ & $1: 640$ & $1: 320$ & 0 & 0 & 0 & 0 & 0 & $1: 20$ & 0 & $1: 40$ \\
27 & $1: 5,120$ & $1: 640$ & $1: 1,280$ & 0 & $1: 80$ & 0 & 0 & 0 & 0 & $1: 20$ & 0 \\
28 & $1: 5,120$ & $1: 2,560$ & $1: 2,560$ & $1: 640$ & 0 & $1: 640$ & 0 & $1: 160$ & 0 & 0 & 0 \\
29 & $1: 5,120$ & $1: 320$ & $1: 320$ & $1: 160$ & 0 & $1: 80$ & 0 & $1: 80$ & 0 & 0 & 0 \\
30 & $1: 640$ & $1: 160$ & $1: 160$ & $1: 20$ & 0 & 0 & 0 & 0 & 0 & 0 & 0 \\
\hline
\end{tabular}

AB13 = "typical strain" streptococcus; Sc. Fv. = scarlet fever streptococcus; Throat $=$ hemolytic streptococci isolated from the throats of various individuals; Tonsil = hemolytic streptococcus isolated from an excised tonsil; Tooth $=$ hemolytic streptococcus isolated from a root canal of a tooth; Stool = hemolytic streptococcus recovered from a stool specimen; Gastric ulcer = alpha prime type of streptococcus isolated from a gastric ulcer by E. W. Saunders (2). 
as 1:640 with the serum of one of the patients with chronic infectious arthritis, while the others showed slight or no agglutination. It seems probable that if the series had been larger, several other strains would have agglutinated well with the serums of patients with chronic infectious arthritis. The observations from these agglutinations were quite compatible with those reported by Stevens and Dochez (3), who performed agglutination tests with immune serums against scarlet fever, erysipelas, and pyogenic streptococci, and found that some of the pyogenic streptococci had antigenic properties in common with the hemolytic streptococci from scarlet fever and erysipelas.

\section{SUMMARY AND DISCUSSION}

The serums of patients with chronic infectious arthritis usually gave strong agglutination reactions with "typical strain" streptococci (93.6 per cent in this series). This phenomenon is not observed with control serums with the possible exception of the serums from patients with acute tonsillitis and acute bronchitis. The close association of upper respiratory infection with chronic infectious arthritis has already been mentioned.

In marked contrast to these strong agglutinative properties in the serums of patients with chronic infectious arthritis is the slight or negative agglutinin content of the serums of patients with degenerative arthritis. This biologic distinction between the two types of arthritis offers strong evidence in support of the theory of their diverse etiologies-indeed, the contrast is so definite that it might well be used clinically in the differential diagnosis of the two most common chronic infections of the joints.

The serums of 3 patients with chronic polyarthritis following rheumatic fever failed to give positive agglutination reactions with "typical strains." This would seem to indicate that chronic polyarthritis following rheumatic fever and primary chronic infectious arthritis are not etiologically identical, although clinically they present similar pictures.

Three patients whose serums gave strong agglutination reactions during the course of the disease were found to have completely lost their agglutinins following recovery. In the opinion of the writers, 
these observations lend strong support to the theory that the "typical strain" streptococcus is an important etiologic factor in chronic infectious arthritis.

The demonstration of streptococcal agglutinins in the joint fluids of 3 cases of chronic infectious arthritis was particularly interesting in view of the fact that in a previous study we had succeeded in recovering "typical strains" of streptococci in joint cultures from such patients. This is analagous to the high agglutinin content of empyema fluid in pneumococcal pneumonia.

A Streptococcus hemolyticus from a case of scarlet fever and a Streptococcus hemolyticus from a case of erysipelas have been agglutinated by the serums of arthritic patients to almost as high a titer as have "typical strain" streptococci. Stevens and Dochez (3) have shown that scarlet fever streptococci and erysipelas streptococci, as well as the individual strains from each disease, are not all biologically identical, but that those strains that are not identical usually have antigenic properties in common. It would appear probable, then, that if a larger number of strains from scarlet fever and erysipelas patients had been studied in this investigation, a certain number of them would have given strongly positive agglutination reactions with arthritic patients' serums, while others would have given weakly positive or negative agglutination reactions.

Two hemolytic streptococci from the throats of patients free from any evidence of arthritis, scarlet fever, or erysipelas gave agglutination reactions as high as 1:640 with the serum from a patient with chronic infectious arthritis, while the other hemolytic streptococci gave little or no agglutination. It seems reasonable to conclude that the "typical strain" streptococci have antigenic properties in common with the hemolytic streptococci from patients with scarlet fever and erysipelas, as well as with some hemolytic streptococci from other sources. Such an admission, however, in no wise weakens the confidence of the writers that the agglutinins present in the serums of patients with chronic infectious arthritis are specific agglutinins differing in no essential respect from the specific agglutinins which accompany infections with the typhoid bacillus, the pneumococcus, or any other pathogenic micro-organisms. 


\section{CONCLUSIONS}

1. The serums of patients with chronic infectious arthritis usually give a strong specific agglutination reaction with "typical strains" of streptococci recoverable from the blood and joints of patients with this disease. Control serums do not show this reaction.

2. Chronic infectious arthritis can be differentiated from degenerative arthritis and from chronic polyarthritis following rheumatic fever by the agglutination reaction. The agglutination reactions suggest different etiologies for the three forms of arthritis.

3. A close antigenic relationship between "typical strain" streptococci and the hemolytic streptococci from scarlet fever and erysipelas is established.

\section{BIBLIOGRAPHY}

1. Cecil, R. L., Nicholls, E. E., and Stainsby, W. J., Arch. Int. Med., 1929, xliii, 571. The Bacteriology of the Blood and Joints in Chronic Infectious Arthritis.

2. Saunders, E. W., Arch. Int. Med., 1930, xlv, 347. The Serologic and Etiologic Specificity of the Alpha Streptococcus of Gastric Ulcer.

3. Stevens, F. A., and Dochez, A. R., J. Exp. Med., 1926, xliii, 379. Studies on the Biology of Streptococcus. V. Antigenic Relationships Between Strains of Streptococcus from Scarlet Fever and Erysipelas. 